\title{
Endoscope-assisted strip craniectomy and postoperative helmet therapy for treatment of craniosynostosis
}

\author{
John Berry-Candelario, M.D., M.P.H., ${ }^{1}$ Emily B. Ridgway, M.D., ${ }^{2}$ \\ Ronald T. Grondin, M.D., ${ }^{1}$ Gary F. Rogers, M.D., J.D., M.B.A., ${ }^{2}$ \\ and Mark R. Proctor, M.D. 1 \\ Departments of ${ }^{1}$ Neurological Surgery and ${ }^{2}$ Plastic and Reconstructive Surgery, Children's Hospital Boston, \\ Harvard Medical School, Boston, Massachusetts
}

\begin{abstract}
Object. The primary goals of treatment in the infant with craniosynostosis are to correct the deformity and allow for adequate brain growth in as safe and effective a manner as possible. Herein, the authors present the results of treating craniosynostosis using an endoscope-assisted strip craniectomy and postoperative helmet therapy (EASC + PHT) in the hopes of providing further evidence of its role in the treatment of multiple different forms of craniosynostosis. This is a retrospective review of the patients treated with this technique at Children's Hospital Boston.

Methods. The electronic medical records of all children with craniosynostosis treated using this technique were reviewed retrospectively. A priori, data were collected for deformity type, patient age at surgery, number of transfusions, operative time, length of hospital stay, and anthropometric measurements.

Results. One hundred seventy-three patients (61 females and 112 males) were treated at our institution between July 2004 and March 2011 with EASC + PHT. The mean operative time was 46.30 minutes. Eight (4.6\%) of the 173 patients received blood transfusions. The average length of hospital stay was 1.35 days, with the majority of patients being discharged the day after surgery. All complications and any patient who required additional craniofacial reconstructions are discussed. In addition, a subgroup analysis was done for patients who had undergone surgery and had longer than 1 year of follow-up.

Conclusions. The authors' growing database of patients supports the experiences described by others that early treatment of craniosynostosis with an EASC + PHT is a safe and efficacious technique. In addition, cost reduction due to decreased hospital stay and limitation of blood transfusions are demonstrable benefits associated with the use of this technique. (DOI: 10.3171/2011.6.FOCUS1198)
\end{abstract}

\section{KEY WORDS • craniosynostosis • endoscopic surgery $\bullet$ helmet therapy
strip craniectomy}

$\mathrm{T}$ He premature fusion of calvarial sutures, better known as craniosynostosis, is a well-known cause of deformational changes to the skull. It has an estimated frequency of 0.4 of 1000 live births, with the majority consisting of isolated single suture defects. ${ }^{6}$ The etiology of most cases is sporadic and not well established, although there is a strong genetic component and more than 70 identified syndromes. Early scientific inquiry into this disease entity revealed several theories as to its pathogenesis. Virchow ${ }^{22}$ first described the fundamental aberrant growth patterns, noting the "compensatory growth" of the calvaria. Moss ${ }^{13}$ espoused the "fundamental matrix theory," which stated that the active growth of

\footnotetext{
Abbreviations used in this paper: EASC + PHT = endoscopeassisted strip craniectomy and postoperative helmet therapy; FOA $=$ frontoorbital advancement; $\mathrm{HCP}=$ head circumference percentile.
}

the underlying brain dictated the passive cranial growth along the suture lines. These theories, with the presumption that prenatal abnormalities at the fused suture and/or skull base are the cause of the deformity, represents the foundation for early and minimally invasive approaches using endoscope-assisted surgery with postoperative helmet therapies.

The advent of suturectomy began in the late $1800 \mathrm{~s}$ at a time when craniosynostosis had been recently recognized as an entity that could lead to neurological dysfunction and cognitive comorbidities. Many of the surgeries were likely performed for nonsynostotic microcephaly, and there was an alarmingly high mortality rate associated with the surgery, which resulted in it falling out of favor. ${ }^{8}$ This consequence marked the end of surgery for craniosynostosis for more than 30 years. Variations of these suturectomy techniques gradually resurfaced in 


\section{J. Berry-Candelario et al.}

the mid-1900s and became the standard treatment for craniosynostosis for the next half century. The biggest limitation of these methods was that they relied exclusively on the brain growth to effect changes in cranial shape and size. Consequently, outcomes were variable and often limited by early refusion at the craniectomy sites. Attempts to line the craniectomy defect with a foreign material (for example, polyethylene film, Gore-Tex, and other substances) to prevent refusion also failed to succeed.?

In the 1970 s, Tessier ${ }^{20,21}$ introduced pioneering techniques for treatment of craniosynostosis in which large segments of the cranium were removed, remodeled, and stabilized as the situation required. The limitations of suturectomy for advanced disease fueled these developments, along with the discovery by Delashaw and colleagues ${ }^{4}$ that the major cause of the cranial deformity was compensatory overgrowth at adjacent sutures. Thus, the desired changes in shape and volume were established intraoperatively, and the bony segments were fixed to maintain the correction. Because the postoperative cranial result was not dependent on postoperative brain expansion, the outcomes of these operations were more predictable than simple or extended craniectomy procedures, and cranial remodeling became the preferred operation for craniosynostosis. However, these techniques are associated with significant blood loss, lengthy surgical times (3-8 hours) and hospital stays (4-7 days), and often the need for postoperative intensive care unit monitoring. ${ }^{18}$ Surgery is often delayed until the infant is older (6-12 months) to limit operative morbidity, but this allows the deformities to become more severe. The reported complications of the cranial vault remodeling procedures include inadequate calvarial shape correction, improper skull reossification, palpable and visible deformities and asymmetries, loosening of hardware, migration of screws into the brain parenchyma through the dura, extensive blood losses, and problems associated with blood transfusion reactions. ${ }^{718}$ Moreover, many specialists in the field have noted both anecdotally and in published works that even with large repairs, the growth patterns were not normal over time, and there was reversion toward the original dysmorphology. ${ }^{5}$ Therefore, the cosmetic results of larger procedures are certainly not perfect.

In 1999, Barone and Jimenez ${ }^{2}$ published the endoscopic approach for suturectomy followed by orthotic therapy. In the first 10 cases, they noted a significant difference in blood loss and the need for transfusions, shorter operative times and hospital stays, and decreased hospital costs with the endoscopic approach than with conventional cranial vault remodeling. They expanded their indications to include coronal, metopic, and lambdoid synostoses. ${ }^{9,10}$ Other authors have supported the conclusions of Barone and Jimenez. ${ }^{14}$ For those who believe that the era of suturectomy has already been tried and failed, it is important to note the use of newer technologies in achieving the result. The effectiveness of the surgery is not simply in the release operation, but in the subsequent redirection of skull growth through the use of external orthoses, springs, or distractor devices. Technologies have changed, and dismissing the operation because it had a high failure rate in the past is problematic.

This report describes the experience of a single sur- geon (M.R.P.) using EASC + PHT to treat many forms of craniosynostosis. In addition to demonstrating the effect of this operation on cranial shape and volume, we catalog the complications and limitations of this technique.

\section{Methods}

\section{Patient Selection, Preoperative Imaging, and Anesthesia}

Infants diagnosed with craniosynostosis who were younger than 6 months of age were considered for the endoscopic approach. Extended discussions on the risks and benefits of open and endoscopic approaches were undertaken with the family. The minimally invasive approach was considered appropriate for all infants younger than 3 months with single suture synostosis and older infants only if they had mild deformity. At our institution, approximately $50 \%$ of patients are young enough to be offered endoscopic techniques (this percentage has been increasing over time), and most families have elected to undergo this surgery. The remaining patients undergo open surgery at $8-10$ months. We would generally not recommend the endoscopic approach if the child had a significant deformity and was older than 3 months of age. Although there are no absolute guidelines on this, for sagittal synostosis the threshold for endoscopic surgery in a child older than 3 months would generally be a cranial index greater than $0.66-0.68$, also subjectively accounting for the more subtle manifestations. For example, the typical saddle deformity responds very well to endoscopic surgery even at older ages, whereas severe frontal bossing and marked bathrocephaly does not. For other synostoses, where objective criteria are less available, the criteria are more difficult to distinguish on an age-related basis. Very profound trigonocephaly in older children and profound turricephaly in children with bilateral coronal synostosis will likely not respond to endoscopic techniques at older ages. Similarly, profound asymmetry in unilateral coronal synostosis is likely best treated when the patient is younger than 3 months. In addition, some patients with multiple suture synostoses were considered for endoscopic release, either as primary treatment or to prevent significant progressive compensatory deformity, with the expectation that another surgery might still be required in the future (that is, to prevent the development of severe turribrachycephaly in children with bilateral coronal synostosis). All infants had routine laboratory samples drawn, and blood was crossmatched and available in the operating room at the start of the case. Computed tomography scans were not routinely obtained for single suture synostosis, but they were acquired when it was thought necessary to establish the diagnosis or guide surgical decisions. For example, we would not perform endoscopic surgery in a child with multisuture synostosis and multiple bony erosions or irregularities on the endocortical surface, as separating the dura from the bone becomes far more complex. In general, endoscopic techniques are feasible because the dura easily separates from the bone at a fused suture, and if preoperative data would indicate that separation is not likely, one should probably not perform the case in a minimally invasive fashion.

General endotracheal anesthesia was used, and 2 


\section{Endoscope-assisted strip craniectomy}

intravenous catheter lines were inserted after induction. Early in the series arterial lines were common, but as the technique evolved, they were rarely used. No central lines were used. Precordial Doppler ultrasonography was used in all cases to assess for air embolism.

\section{Positioning and Skin Preparation}

For sagittal synostosis, the patients were placed prone using a specialized head holder (DORO cranial stabilization, Promed Instruments; Fig. 1) that kept the head extended, with the torso on chest rolls. For metopic or coronal sutures, the patients were supine, and the head was placed on a horseshoe headholder. For lambdoid synostosis, the patient was kept prone with the head supported on a horseshoe headholder. The scalp was prepared with povidone-iodine solution, and the area of intended incision was infused with Marcaine $0.25 \%$ and epinephrine $1 / 200,000$.

\section{Incision and Subgaleal Dissection}

The incisions were generally $1.5-2.5 \mathrm{~cm}$ in length and all oriented perpendicular to the involved suture but varied in number and location by suture type as follows: sagittal, 2 incisions in the midline, one just posterior to the coronal sutures and the other at the junction of the lambdoids; metopic, 1 midline incision just posterior to the hairline; unilateral and bilateral coronal, 1 incision at the midsuture(s); and lambdoid, 2 incisions, one at the region of lambda and the other at the inferior end of the suture.

\section{Initial Craniectomy and Dural Dissection}

After exposure of the suture, a bur hole was placed directly over the affected suture. A Kerrison rongeur was used to increase the diameter of the bur hole to $1 \mathrm{~cm}$. The periosteum was elevated off the overlying skull along the direction of the suture. Finally, the endoscope was inserted through the bur hole and the dura was dissected from the overlying skull under direct visualization. The endoscope was used in all cases. In some instances, it did not provide better visualization than direct inspection with loupes, but it afforded excellent light and allowed the surgeon to transilluminate and see how far the dissection had

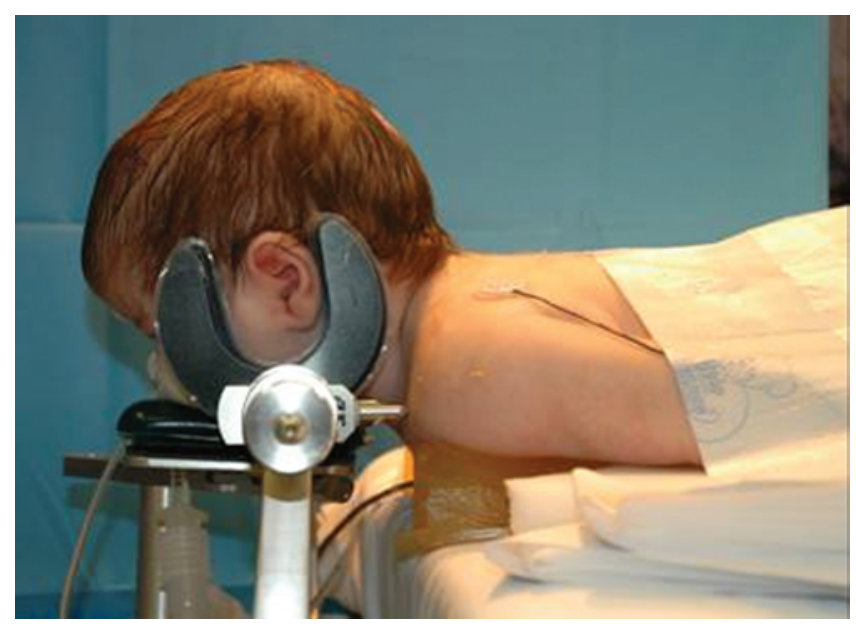

FIG. 1. Photograph of patient positioned in the DORO head holder. proceeded when performed in an operating room with the ambient lights turned off. Since the dura is generally not adherent at a fused suture, dissection was usually done in a blunt fashion with the endoscope and suction. Emissary veins were coagulated with bipolar cautery and divided. Heavy scissors were then used to cut a 1- to 2-cm-wide strip of bone centered on the affected suture, and the bone was removed. (Early in the series, wider craniectomies were performed, as well as barrel staving, but this was believed to be unnecessary to achieve good results, and bone removal has been minimized. Using a cadre of objective and subjective measures, we did not find that a wider craniectomy improved results. In addition, a few patients who received barrel staves developed bone callus at the regions that were cut; therefore, this technique was discontinued.) The callus regions remodeled over about 2 years. The wounds were irrigated, and Gelfoam (Pfizer, Inc.) was placed over the craniectomy site. Bone edges were not coagulated. The galea and skin were closed in layers with absorbable sutures. A pressure dressing along the craniectomy was applied for 12 hours.

\section{Cranial Remolding Helmets}

Within 1 week of the procedure, a cranial molding helmet was fitted for the child. In our opinion, the design and subsequent modifications of the helmet were critical to the success of this procedure. One significant advantage of the helmet, compared with other technologies such as springs and distractors, is the ability to modify the skull growth in 3 dimensions and to be adjustable over time in all dimensions in reaction to actual skull growth. Thus, there was close follow-up by an experienced certified orthotist and the craniofacial team. In general, each helmet was designed and contoured to contact all areas of the infant's cranium except where growth was desirable. Good orthotic fitting requires conceptualization of how cranial growth in specified areas will correct the deformity over time, and there can be a steep learning curve. Since the shape changes are most pronounced early in treatment, careful assessment and thoughtful adjustments were necessary during the first 3-4 months. The helmet was discontinued once the desired phenotype was obtained or when the infant reached 1 year of age. The cost of the orthosis was, on average, \$2200/helmet, including design and all subsequent adjustments. Children needed either 1 or 2 helmets during the treatment period. In a separate detailed analysis of costs, even adjusting for cost of helmets and time lost from work for the families, we have seen a substantial cost reduction using the minimally invasive techniques. Comparing 10 patients treated with minimally invasive techniques and 10 patients treated with open reconstruction, the total costs for endoscopic techniques averaged $42 \%$ of the costs for open techniques. ${ }^{1}$

\section{Follow-Up}

As a rule, all of our patients are monitored until 6 years of age, when brain growth is essentially complete. This is true for patients treated endoscopically and by open techniques. In the results, we list separately the overall group and those patients who have had more than 1 year 
of follow-up since completion of treatment (surgery and helmeting).

\section{Results}

Between July 2004 and March 2011, 173 patients were treated with endoscopic strip craniectomy by the senior author (M.R.P.). There were 61 girls and 112 boys. The frequency of involved suture(s) in descending order was as follows: sagittal, 55.5\% (96 patients); unilateral coronal, $15.0 \%$ (26 patients); metopic, $13.9 \%$ (24 patients); bilateral coronal, $6.9 \%$ (12 patients); multisuture other than bicoronal, 6.4\% (11 patients); and lambdoid, $2.3 \%$ (4 patients) (Table 1). The average age at operation was 2.9 months (range 0.79-7.75 months), with a median age at operation of 2.89 months. The mean operative time was 46.30 minutes (range 25.0-93.0 minutes). The mean length of hospital stay was 1.35 days (range 1-3 days, with 1 outlier at 23 days; this patient had multisuture synostosis and significant cardiac abnormalities and remained in the hospital for comorbidities). Three patients with comorbid conditions required an overnight stay in the intensive care unit. Eight patients (4.6\%) required perioperative blood transfusions. No absolute hematocrit or hemoglobin nadir threshold dictated blood transfusion; however, in the setting of hemodynamic instability and decreasing hemoglobin and/or visible blood loss, a blood transfusion was provided. The nadir was a hematocrit of $20 \%$. There were no deaths. The mean follow-up from the operative date was 3.99 years (Table 2).

In addition, we completed an in-depth analysis of the series of the 111 patients who underwent at least 1 year of follow-up, having thereby completing the treatment protocol. This group of 111 is the subset that was closely analyzed below. Among this subset, the PHT was typically completed in a mean of $7.78 \pm 2.55$ months (range 2.37-21.55 months).

\section{Patient Outcomes Varied by Fusion Type}

Sagittal Synostosis. A total of 61 patients in this group completed helmet therapy and have at least 1 year of follow-up, and all 96 patients are monitored with regular follow-up. The mean duration of helmet therapy was 7.47 months. Patients experienced a $36.85 \%$ increase in HCP. Cranial index, as measured by a single observer in all cases (senior author [M.R.P.]), increased from a mean

TABLE 1: Distribution data in patients who underwent treatment of craniosynostosis

\begin{tabular}{cc}
\hline Type of Synostosis & No. of Patients (\%) \\
\hline sagittal & $96(55.5)$ \\
metopic & $24(13.9)$ \\
unilat coronal & $26(15.0)$ \\
bicoronal & $12(6.9)$ \\
lambdoid & $4(2.3)$ \\
multisuture & $11(6.4)$ \\
total & $173(100)$ \\
\hline
\end{tabular}

TABLE 2: Hospital course data in 173 patients*

\begin{tabular}{ll}
\hline \multicolumn{1}{c}{ Parameter } & \multicolumn{1}{c}{ Value } \\
\hline mean op duration in mins (range) & $46.30(25.0-93.0)$ \\
no. of transfusions (\%) & $8(4.6)$ \\
mean LOS in days (range) & $1.35(1.0-3.0) \dagger$ \\
mean FU length from time of op in yrs (range) & $3.99(1.76-6.66)$ \\
\hline${ }^{*}$ FU = follow-up; LOS = length of stay. \\
$\dagger$ The value was calculated without the one outlier in the multisuture \\
group with cardiac anomalies.
\end{tabular}

of $0.69 \pm 0.04$ preoperatively to $0.77 \pm 0.04$ at the 1 -year follow-up and regressed to and stabilized at $0.76 \pm 0.03$ for patients observed longer than 2 years (Fig. 2). Multivariate regression analysis demonstrated that age at surgery $(p=0.0005)$ was an independent variable for change in cranial index and increase in HCP. Overall, there was uniformly excellent cosmetic correction (Table 3). Complications included 1 incisional abscess requiring admission for incision and drainage but no long-term antibiotics and 1 sagittal suture refusion and 2 cases of additional suture fusion, all 3 of which required open cranial vault remodeling. Among those patients still undergoing helmet therapy, there were no complications, and surveillance of follow-up data indicates similar improvements in cranial index and HCP as those patients who have completed therapy.

Unilateral Coronal Synostosis. Twenty patients completed helmet therapy and have longer than 1 year of follow-up, and all 26 patients are monitored with regular follow-up. Eleven of the patients had a left-sided fusion. Helmet therapy lasted an average of 9.19 months. Patients experienced a $12.14 \%$ mean increase in HCP. Cranial asymmetry decreased from a mean of $8 \mathrm{~mm}$ (range 6-10 $\mathrm{mm}$ ) to $3.7 \mathrm{~mm}$ (range 1-6 mm) (Fig. 3). There was excellent correction of nasal root deviation, facial asymmetry, and orbital dystopia with a trend toward reduced associated ophthalmological findings (for example, astigmatism and ocular torticollis) $)^{5}$ and good to excellent improve-
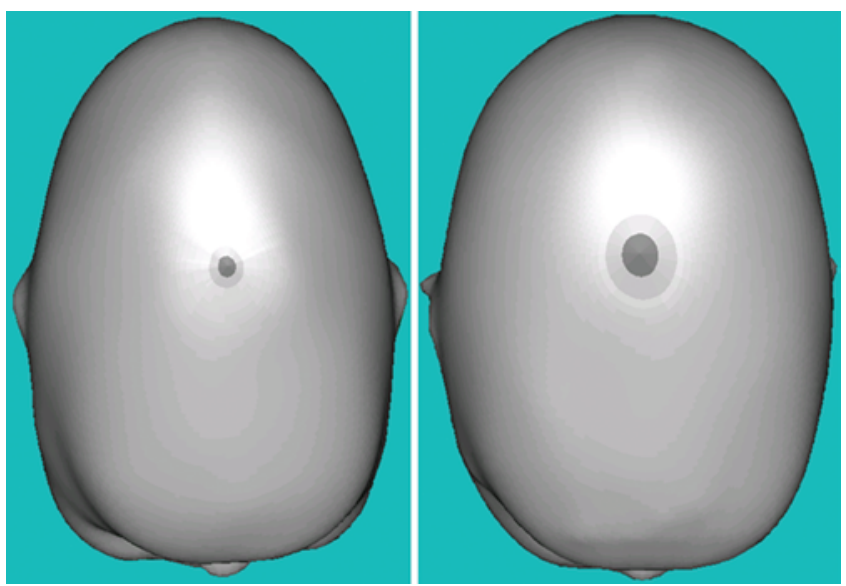

FIG. 2. Preorthotic (left) and postorthotic (right) laser scanning report documenting the change in head shape and improvement in cranial index in an infant with sagittal synostosis. 


\section{Endoscope-assisted strip craniectomy}

TABLE 3: Postoperative clinic outcomes following completion of surgical and helmet therapy*

\begin{tabular}{lccccc}
\hline & \multicolumn{5}{c}{ Mean \pm SD } \\
\cline { 2 - 6 } \multicolumn{1}{c}{ Type of Synostosis } & Length of FU (yrs) & $\begin{array}{c}\text { Length of Helmet } \\
\text { Therapy (mos) }\end{array}$ & Preop HCP & Postop HCP & Change in HCP (\%) \\
\hline bilat coronal (9 patients) & $2.21 \pm 0.79$ & $8.63 \pm 1.58$ & $11.31 \pm 1.14$ & $22.84 \pm 23.27$ & $11.06 \pm 24.58$ \\
lambdoid (3 patients) & $1.86 \pm 1.61$ & $5.43 \pm 1.68$ & $23.33 \pm 20.0$ & $53.17 \pm 46.30$ & $29.83 \pm 27.51$ \\
metopic (11 patients) & $1.01 \pm 0.69$ & $7.20 \pm 2.52$ & $46.45 \pm 25.33$ & $74.21 \pm 25.37$ & $27.75 \pm 19.08$ \\
unilat coronal (20 patients) & $2.09 \pm 1.48$ & $9.19 \pm 4.05$ & $31.14 \pm 27.11$ & $50.56 \pm 28.84$ & $12.14 \pm 17.64$ \\
sagittal (61 patients) & $2.34 \pm 0.94$ & $7.47 \pm 1.93$ & $57.10 \pm 32.16$ & $93.60 \pm 22.06$ & $36.85 \pm 33.23$ \\
total (104 patients) & & & & & \\
\hline
\end{tabular}

* Includes 111 patients who have 1 year of follow-up since completion of treatment. Seven multisuture patients who have completed therapy are not shown.

ment of forehead symmetry. Although the forehead symmetry was slightly inferior to traditional FOA at 1 year, it continued to improve at each consecutive annual visit. We have analyzed the findings in unilateral coronal synostosis using 3D photogrammetry. Comparing children who had undergone endoscopic versus open techniques, we found statistically significant improvement in facial symmetry in the minimally invasive group and nonsignificant differences in forehead symmetry at a mean age of 2.8 years (unpublished data, 2009). Two patients had persistent deformity for which FOA was recommended. Another patient experienced a small dural tear requiring a larger than average craniectomy at the time of surgery and had a persistent skull defect, which required cranioplasty repaired through the original $1.5-\mathrm{cm}$ incision. Three patients (12\%) were diagnosed with Saethre-Chotzen syndrome. One of these patients is currently undergoing helmet therapy. The second patient experienced fusion of the sagittal suture at approximately 2 years of age. He has not developed elevated intracranial pressure and is being observed at this time. The third patient has not had any complications since completing helmet therapy (Table 3).

Bilateral Coronal Synostosis. Nine patients completed helmet therapy, and all 12 patients are monitored with regular follow-up. One patient has been diagnosed with Pfei-
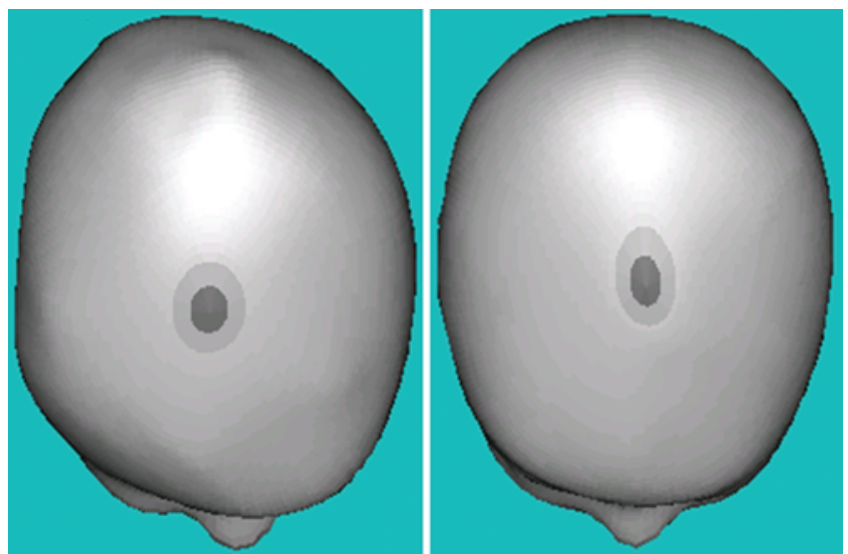

FIG. 3. Preorthotic (left) and postorthotic (right) laser scanning report documenting the change in head shape in an infant with unilateral coronal synostosis. ffer syndrome (FGFRl mutation), and another has been diagnosed with Apert syndrome. For those who have completed treatment, helmet therapy lasted an average of 8.63 months. These patients experienced a mean change in $\mathrm{HCP}$ of $11.06 \%$ and a mean maximum decrease in cranial index of -0.08 (mean preoperative cranial index 0.94 [range $0.84-1.0$ ] and mean postoperative cranial index 0.86 [range 0.81-0.91]) (Fig. 4). Turribrachycephaly was well corrected and forehead projection improved, although the improvement appeared to be less dramatic than with traditional FOA. These are subjective assessments, and we have not correlated them to objective data. Two patients experienced refusion of the coronal sutures, as well as additional sutures, with the sagittal suture in one patient and sagittal and metopic sutures in the other. Both cases were heralded by a falloff in head circumference, and required open cranial vault remodeling. On genetic evaluation, one of the patients was later diagnosed with Saethre-Chotzen syndrome. The genetic workup of the second patient with refusion was negative for TWIST, and FGFR1, 2, and 3. All other patient genetic evaluations were negative (Table 3 ).

Metopic Synostosis. Eleven patients completed helmet therapy, and all 24 patients are monitored with regular follow-up. For those who had completed helmet therapy, the average duration of helmet therapy was 7.20 months. Patients experienced a mean increase of $27.75 \%$ in head circumference profile. The midline ridge was very effectively treated and paramidline ridges, although slow to
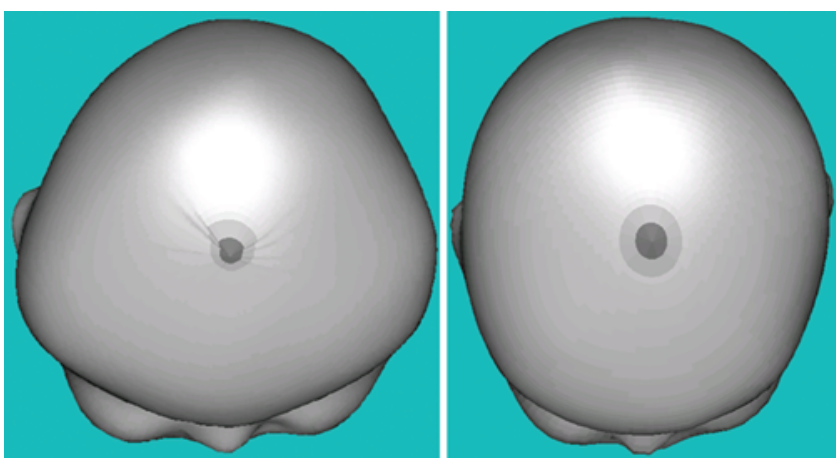

Fig. 4. Preorthotic (left) and postorthotic (right) laser scanning report documenting the change in head shape in an infant with bilateral coronal synostosis. 


\section{J. Berry-Candelario et al.}

resolve, did improve with time (Fig. 5). The correction of superolateral orbital rim retrusion was not consistent in our assessment, and subjectively we believed that FOA offers a superior lateral brow projection. There was no adequate way to document these discrepancies, as we did not routinely obtain images in the child. Interestingly, families tended to be very happy with the results and no revisions were performed or are pending (Table 3). All genetic evaluations were negative. One of the patients developed a postoperative wound infection and was treated with surgical debridement but no long-term antibiotics.

Lambdoid Synostosis. Three patients received helmet therapy that lasted an average of 5.43 months, and all 4 patients are monitored with regular follow-up. Patients experienced a mean increase of $29.83 \%$ in HCP. Cranial asymmetry decreased from a mean of $6.5 \mathrm{~mm}$ (range 6-7 $\mathrm{mm}$ ) to a mean of $2 \mathrm{~mm}$ (range $1-2 \mathrm{~mm}$ ). There was an excellent reduction of the windswept appearance and occipital asymmetry. In our subjective opinion, the results appeared better than those achieved with open posterior calvarial vault remodeling, which often seems inadequate in correcting this windswept appearance. There were no good objective measures to assess the outcomes, but we believed that they more than rivaled the larger operations (Table 3). No revisions were performed or are pending. No Chiari malformations were present.

Multisuture Synostosis. Eleven patients had more than 1 fused suture. Three were unable to complete primary helmet therapy due to refusion of the treated suture (1 patient) and/or fusion of additional sutures (2 patients). In these cases, the reclosure was heralded by a falloff in the HCP. One of these patients was later diagnosed with Crouzon syndrome. Genetic anomalies but no syndromes have been found for the remaining patients who experienced refusion. These 3 patients underwent formal cranial vault expansion/ remodeling. In addition, 1 patient with sagittal and unilateral coronal synostosis had persistent deformity despite no refusion or additional fusion; FOA was recommended. Seven patients had good results and no complications. An eighth patient is currently undergoing helmet therapy and is being monitored with regular follow-up.

\section{Discussion}

This report confirms prior reports that EASC + PHT allows safe, early treatment of craniosynostosis and results in improvement in cranial shape and head circumference in most patients. ${ }^{2,9,10}$ Based on available anthropometric measurements and the subjective assessment of our craniofacial team, the shape correction was excellent in patients with sagittal and lambdoid synostosis, good in those with unilateral and bilateral coronal synostosis, and more variable for metopic synostosis. All patients also had a significant increase in HCP after release.

Our data also support the findings of other authors that the most important determinants of success after EASC + PHT is the age at which the suturectomy is done and the compliance with the PHT. We observed that in the subgroup of patients with sagittal synostosis, improvement in shape and HCP was inversely correlated
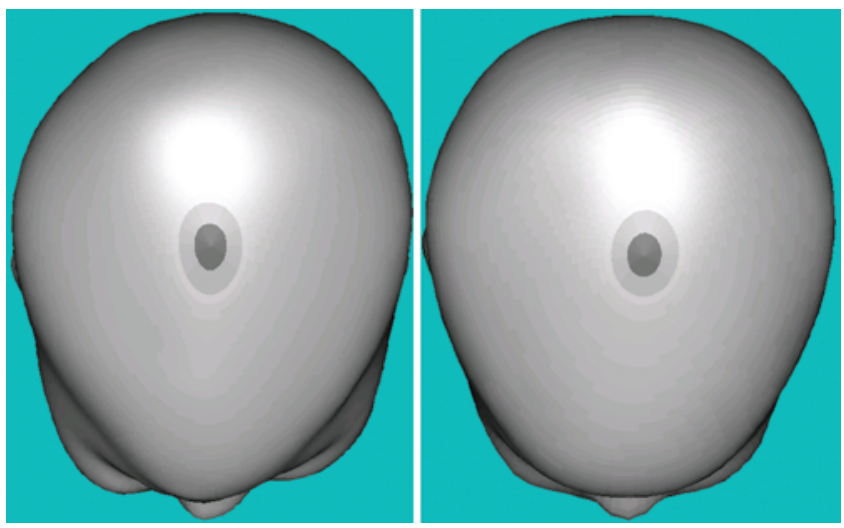

FIG. 5. Preorthotic (left) and postorthotic (right) laser scanning report documents the change in head shape in an infant with metopic synostosis.

with the age at which the procedure was done. This result is not unexpected given the mechanism by which EASC + PHT works. Because correction is achieved by carefully directed 3D brain expansion, the process is more efficient in younger infants who have the fastest rate and greatest potential for cerebral expansion. Ingraham and colleagues $^{7}$ noted that age at the time of suturectomy played a significant role in the long-term outcome. They attributed poor outcomes to either a delay in surgery or surgical procedures that did not adequately free the involved bones or maintain their separation for an adequate length of time.,17 Ingraham et al. ${ }^{7}$ and Shillito and Mat$\operatorname{son}^{18}$ recommended that treatment for synostosis occur at 4-6 weeks of age to allow for maximum growth to occur. Similarly, Jimenez and colleagues ${ }^{9,10}$ reported that their best results were obtained when patients were referred very early in life. Their conclusions were that the best time to perform this procedure is at or before 3 months of age. It is our recommendation that suturectomy be performed as early as possible, balancing factors such as general infant health and weight, but ideally at or slightly before 3 months.

In our view, surgery alone is not adequate, and the other critical component to the success of EASC + PHT is compliant use of a well-designed orthosis. This is philosophically how the procedure is different from the strip craniectomy procedures of years past, and it appears to be a primary determinant of outcome. Although the utility of the helmet after this procedure has been questioned, in our experience, failure to guide the cranial expansion with an external orthotic is a principal reason for poor or under-correction. For the most part, we saw reversion of the deformity if the helmet was discontinued too early and inadequate results in the few children with poor helmet design or compliance. Conversely, a well-designed helmet really allowed for excellent correction in multiple planes of growth. By controlling growth in most areas, the orthotic acts by focusing most or all of the cranial growth in the areas where it is needed. Thus, the brain becomes a very effective internal distractor. Without a guiding orthotic device, expansion occurs in all directions and the correction is less complete. Jimenez and colleagues ${ }^{9,10}$ advocated continuing helmet-molding therapy 
until 1 year of age to prevent reversion. They reported that given cranial growth dynamics, once the patients have obtained normocephaly at 12 months, the shape will be maintained thereafter. ${ }^{9}$ It has been our practice to maintain helmet-molding therapy until 1 year of age or until normocephaly has been achieved and maintained on serial examination. Our data do demonstrate a small regression from maximal cranial index in patients with sagittal synostosis, most noticeably in the 2 nd year of life. Consequently, we now maintain the helmet until the cranial index is greater than 0.8 , or until 1 year of age. Relapse has also been observed in patients with sagittal synostosis patients treated with open cranial vault remodeling. Fearon and colleagues ${ }^{5}$ observed 132 patients for 3-11 years postoperatively (mean follow-up time 4.7 years) and documented initial overcorrection of cephalic index followed by an overall regression of cranial index. They also reported a diminished capacity for head circumference growth; patients had head circumferences significantly above normal preoperatively that decreased to less than 1 standard deviation over the mean. They concluded that there is an overall deficiency in skull growth either as a result of the operative intervention or from inherent osseous undergrowth related to the craniosynostosis. The very slight relapse we observed in our patients suggests that the former explanation is more accurate.

One advantage of early treatment with suturectomy is that it prevents secondary deformity. Numerous investigators have demonstrated that many of the characteristic features associated with craniosynostosis occur because the growth of distant patent sutures is affected by the cranial fusion..$^{16,17,19}$ For example, patients with bilateral coronal synostosis often develop secondary turribrachycephaly in early infancy in response to limited anterior cranial expansion. Once established, this can be very difficult to treat surgically. Some groups now recommend performing an early posterior release in children with bilateral coronal synostosis simply to avoid the development of turribrachycephaly (unpublished data, 2009). In our experience, early suturectomy in patients with bilateral coronal synostosis prevents the development of significant turribrachycephaly as the brain can expand anteriorly.

In addition to preventing progressive secondary deformity, we have also noted that early release can also allow normalization of facial and orbital asymmetry in patients with unilateral coronal synostosis. Whereas frontoorbital advancement improves forehead and anterior orbital asymmetry, this procedure does little to improve facial asymmetry or ocular abnormalities inherent in this condition. Oh and coworkers ${ }^{15}$ used 3D photogrammetry to document persistent facial asymmetry in older patients (mean age 14 years) with unilateral coronal synostosis who had undergone frontoorbital advancement in infancy. Becker and colleagues $^{3}$ reported similar findings using serial CT scans. Thus, the correction of frontofacial deformity in patients managed with FOA is inherently incomplete. This realization was in large part responsible for our early willingness to consider EASC + PHT. Impressed by the substantial facial symmetry in patients with unilateral coronal synostosis who were treated with EASC + PHT by Jimenez and colleagues, ${ }^{2,9,10}$ we began to offer this technique to patients with unilateral coronal synostosis in 2004. Consistent with our current protocol, families were advised of the possible need for conventional open cranial remodeling procedure in the event that EASC + PHT failed to produce the desired outcome. However, in a retrospective review using objective and subjective measurements, our observations of the original results of Jimenez and colleagues were correct. Using 3D photogrammetry, we recently demonstrated that EASC + PHT and FOA resulted in similar forehead correction, but the former technique yielded markedly better mid- and lower facial symmetry (see Results, Unilateral Coronal Synostosis). Similarly, we found that children with unilateral coronal synostosis managed with EASC + PHT had significantly less severe V-pattern strabismus and excyclotorsion than age-matched controls who had undergone FOA. Not a single patient in our endoscopic group has required strabismus surgery, whereas almost $60 \%$ of children with unilateral coronal synostosis undergoing standard FOA have persistent strabismus for which surgery is recommended. Therefore, even in the unlikely event that early release failed to gain acceptable cosmetic correction and a secondary open procedure was required, the marked reduction in ocular findings and improved facial symmetry argue for considering this technique. ${ }^{12}$

Like any operative or medical treatment, EASC + PHT can fail if the management is poorly executed. This is well illustrated by the case report of Kohan et al., ${ }^{11}$ wherein they described a pair of twins with sagittal synostosis, one managed by reverse pi procedure and the other by endoscopic release with helmet therapy. They found a posttreatment cranial index of 0.77 in the child managed with cranial vault remodeling and a posttreatment cranial index of 0.63 in the child managed by EASC + PHT. ${ }^{11}$ Although the authors chose to interpret this isolated example as evidence that the EASC + PHT procedure does not work, our uniformly good results with this procedure in patients with sagittal synostosis, as well as many other reports found in the literature, would suggest that this unfortunate outcome was more likely the result of inadequate surgery, poor compliance with helmet therapy, or inadequate duration of helmet therapy. We ascribe the success of our program to careful and consistent follow-up of the patients and very close collaboration and communication with the orthotist. It is particularly important to ensure that the orthotist understands which areas of the calvaria are to be contained (that is, limit growth) and which areas are to be allowed to grow freely. The location and extent of the release will largely determine the success of the correction. During the first years of our program, we routinely saw patients every 2-3 weeks postoperatively to ensure that the orthotic was fitted and contoured appropriately. As our orthotists became more experienced, the frequency was relaxed. In our opinion, lack of attention to the postoperative orthotic management is the main reason why some surgeons report seeing patients with poor correction following this procedure. Regarding the case report by Kohan et al.,"1 anecdotally, our group has treated siblings with sagittal synostosis, one treated with open surgery and the other treated 3 years later with endoscopic surgery followed by helmet therapy. Although both had an acceptable outcome, the cranial index results were better in the child treated with EASC + 


\section{J. Berry-Candelario et al.}

PHT and the family far preferred the minimally invasive treatment. Given the limited conclusions that can be drawn from such an isolated comparison, we elected not to report this.

\section{Limitations of the Study}

Similar to essentially all available studies of surgical technique in the craniofacial literature, our study is limited by it being a retrospective review of a technique with no control group. There was no attempt at randomization, especially as we became more convinced of the efficacy and safety of the minimally invasive techniques. Although we can examine historical controls within our group and the literature, we do not have a direct control group, nor does any other study in the literature. Moreover, the literature and the field are hampered by the lack of objective data by which to interpret and compare results. Even the most objective of our data points, head circumference and cranial index, are often considered poor surrogates by others in the field. In fact, many senior craniofacial experts have argued at national and international meetings that the results in craniofacial surgery are subjective and should not be held to objective standards, and clearly the field is limited by this lack of a firm benchmark. Where available, including the $3 \mathrm{D}$ photogrammetry study and the formal ocular studies for unilateral coronal synostosis, we have tried to objectify the data in a fairly subjective field.

\section{Conclusions}

As technology has evolved, minimally invasive procedures have grown in popularity in all surgical fields. Gall bladders and colons are removed via laparoscopy, cerebral aneurysms are treated via transarterial embolization, and robots are used in fields such as urology. It is therefore no surprise that the field of craniofacial surgery has seen a similar evolution. For any of these technologies to be adopted, the minimally invasive techniques need to show similar safety and efficacy profiles to the standard open procedures. Not surprisingly, there is often reluctance to accept the newer technologies as equivalent or superior. Our experience with EASC + PHT demonstrates the overall safely and efficacy of this treatment. The treatment is inherently different from what many craniofacial experts consider the failed era of strip craniectomy surgery, because newer technologies substantially change the skull growth patterns after surgery, and refusion of the skull before the correction is achieved is extremely unlikely. In our opinion, and based on a series of objective and subjective criteria, for single suture synostosis, the results can rival or exceed those produced by larger open cranial expansion techniques. The need for revision surgery due to refusion of the operated suture or fusion of other adjacent sutures was higher in children with multisuture synostosis. Failure of shape to improve or a sustained decrease in HCP should alert the physician to this possibility and additional CT scanning is advised. Overall, minimally invasive endoscopic suturectomy offers an excellent alternative to traditional open approaches and should be considered an option for children diagnosed prior to 3 months of age.

\section{Disclosure}

The authors report no conflict of interest concerning the materials or methods used in this study or the findings specified in this paper.

Author contributions to the study and manuscript preparation include the following. Conception and design: all authors. Acquisition of data: all authors. Analysis and interpretation of data: all authors. Drafting the article: all authors. Critically revising the article: Proctor, Berry-Candelario, Ridgway, Rogers. Reviewed submitted version of manuscript: Proctor, Berry-Candelario. Approved the final version of the manuscript on behalf of all authors: Proctor. Statistical analysis: Proctor, Berry-Candelario, Ridgway, Rogers. Administrative/technical/material support: Proctor. Study supervision: Proctor, Berry-Candelario.

\section{References}

1. Abbot MM, Roger GF, Proctor MR, Busa K, Meara J: The cost of treating sagittal synostosis in the first year of life: endoscopically-assisted suturectomy and postoperative helmet therapy vs open cranial vault remodeling. J Cranio Surg [in press]

2. Barone CM, Jimenez DF: Endoscopic craniectomy for early correction of craniosynostosis. Plast Reconstr Surg 104: 1965-1975, 1999

3. Becker DB, Fundakowski CE, Govier DP, Deleon VB, Marsh JL, Kane AA: Long-term osseous morphologic outcome of surgically treated unilateral coronal craniosynostosis. Plast Reconstr Surg 117:929-935, 2006

4. Delashaw JB, Persing JA, Broaddus WC, Jane JA: Cranial vault growth in craniosynostosis. J Neurosurg 70:159-165, 1989

5. Fearon JA, McLaughlin EB, Kolar JC: Sagittal craniosynostosis: surgical outcomes and long-term growth. Plast Reconstr Surg 117:532-541, 2006

6. Hunter AG, Rudd NL: Craniosynostosis. I. Sagittal synostosis: its genetics and associated clinical findings in 214 patients who lacked involvement of the coronal suture(s). Teratology 14:185-193, 1976

7. Ingraham FD, Alexander EJ Jr, Matson DD: Clinical studies in craniosynostosis analysis of 50 cases and description of a method of surgical treatment. Surgery 24:518-541, 1948

8. Jacobi A: Non nocere. Med Rec 45:609-618, 1894

9. Jimenez DF, Barone CM: Early treatment of anterior calvarial craniosynostosis using endoscopic-assisted minimally invasive techniques. Childs Nerv Syst 23:1411-1419, 2007

10. Jimenez DF, Barone CM, Cartwright CC, Baker L: Early management of craniosynostosis using endoscopic-assisted strip craniectomies and cranial orthotic molding therapy. Pediatrics 110:97-104, 2002

11. Kohan E, Wexler A, Cahan L, Kawamoto HK, Katchikian H, Bradley JP: Sagittal synostotic twins: reverse pi procedure for scaphocephaly correction gives superior result compared to endoscopic repair followed by helmet therapy. J Craniofac Surg 19:1453-1458, 2008

12. MacKinnon S, Rogers GF, Gregas M, Proctor MR, Mulliken JB, Dagi LR: Treatment of unilateral coronal synostosis by endoscopic strip craniectomy or fronto-orbital advancement: ophthalmologic findings. J AAPOS 13:155-160, 2009

13. Moss ML: The pathogenesis of premature cranial synostosis in man. Acta Anat (Basel) 37:351-370, 1959

14. Murad GJ, Clayman M, Seagle MB, White S, Perkins LA, Pincus DW: Endoscopic-assisted repair of craniosynostosis. Neurosurg Focus 19(6):E6, 2005

15. Oh AK, Wong J, Ohta E, Rogers GF, Deutsch CK, Mulliken JB: Facial asymmetry in unilateral coronal synostosis: longterm results after fronto-orbital advancement. Plast Reconstr Surg 121:545-562, 2008

16. Persing JA, Lettieri JT, Cronin AJ, Wolcott WP, Singh V, Morgan E: Craniofacial suture stenosis: morphologic effects. Plast Reconstr Surg 88:563-573, 1991 


\section{Endoscope-assisted strip craniectomy}

17. Rogers GF, Greene AK, Oh AK, Robson C, Mulliken JB: Zygomaticotemporal synostosis: a rare cause of progressive facial asymmetry. Cleft Palate Craniofac J 44:106-111, 2007

18. Shillito J Jr, Matson DD: Craniosynostosis: a review of 519 surgical patients. Pediatrics 41:829-853, 1968

19. Sullivan SR, Greene AK, Mulliken JB, Rogers GF: Zygomaticotemporal suture synostosis causes progressive facial deformity and asymmetry. Plast Reconstr Surg 123:146e-147e, 2009

20. Tessier P: The definitive plastic surgical treatment of the severe facial deformities of craniofacial dysostosis. Crouzon's and Apert's diseases. Plast Reconstr Surg 48:419-442, 1971

21. Tessier P: Relationship of craniostenoses to craniofacial dys- ostoses, and to faciostenoses: a study with therapeutic implications. Plast Reconstr Surg 48:224-237, 1971

22. Virchow R: Uber den Cretinismus, namentlich in Franken, und uber pathologische Schadelformen. Verh Phys Med Gesell Wurzburg 2:230-271, 1851

Manuscript submitted April 18, 2011.

Accepted June 14, 2011.

Address correspondence to: Mark R. Proctor, M.D., Department of Neurosurgery, Children's Hospital Boston, 300 Longwood Avenue, Hunnewell 2, Boston, Massachusetts 02115. email: mark. proctor@childrens.harvard.edu. 